\title{
Visual search is slowed when visuospatial working memory is occupied
}

\author{
GEOFFREY F. WOODMAN \\ Vanderbilt University, Nashville, Tennessee \\ and \\ STEVEN J. LUCK \\ University of Iowa, Iowa City, Iowa
}

\begin{abstract}
Visual working memory plays a central role in most models of visual search. However, a recent study showed that search efficiency was not impaired when working memory was filled to capacity by a concurrent object memory task (Woodman, Vogel, \& Luck, 2001). Objects and locations may be stored in separate working memory subsystems, and it is plausible that visual search relies on the spatial subsystem, but not on the object subsystem. In the present study, we sought to determine whether maintaining spatial information in visual working memory impairs the efficiency of a concurrent visual search task. Visual search efficiency and spatial memory accuracy were both impaired when the search and the memory tasks were performed concurrently, as compared with when the tasks were performed separately. These findings suggest that common mechanisms are used to process information during difficult visual search tasks and to maintain spatial information in working memory.
\end{abstract}

Natural visual scenes typically contain many objects, and this leads to computational problems that are absent when objects are presented in isolation. To study how complex scenes are processed under controlled laboratory conditions, cognitive scientists have conducted an enormous number of experiments using visual search tasks (for a review, see Wolfe, 1998), and several models of attention have been proposed to account for how information is selectively processed during visual search (e.g., Bundesen, 1990; Cave \& Bichot, 1999; Desimone \& Duncan, 1995; Duncan \& Humphreys, 1989; Luck, Girelli, McDermott, \& Ford, 1997; Treisman, 1988; Treisman \& Gelade, 1980; Treisman \& Sato, 1990; Wolfe, 1994; Wolfe, Cave, \& Franzel, 1989). Almost all models that account for visual search data propose that working memory plays an important role in enabling efficient processing of search arrays. For example, a subset of attention models posit that a template of the search target is maintained in visual working memory, which biases perceptual mechanisms to process items that contain target features (Bundesen, 1990; Desimone \& Duncan, 1995; Duncan \& Humphreys, 1989). In addition, Treisman and colleagues have proposed that once an item is

This study was made possible by individual National Research Service Award F31MH12995-01 from the National Institutes of Health to G.F.W. and by Grants R01 MH63001 and R01 MH65034 from the National Institute of Mental Health to S.J.L. We thank Elsie Braun for help with data collection. Correspondence concerning this article should be addressed to G.F. Woodman, Department of Psychology, Vanderbilt University, Wilson Hall, 11121 st Avenue South, Nashville, TN 372401103 (e-mail: geoffrey.f.woodman@ vanderbilt.edu). selected by attention, an object file of it is created (Kahneman, Treisman, \& Gibbs, 1992; Treisman, 1988; Treisman \& Gelade, 1980; Treisman \& Sato, 1990); object files are temporary object representations that can later be updated, so the creation of an object file may be identical to the creation of a working memory representation. Thus, many influential theories of attention propose that visual working memory representations play an important role in visual search.

To test such proposals, Woodman, Vogel, and Luck (2001) used a dual-task paradigm in which a visual search task was performed during the retention interval of a visual working memory task. ${ }^{1}$ Performance in this dual-task condition was compared with performance when the search and the memory tasks were performed individually. In the visual working memory task, subjects were shown a sample array containing four colored objects, and then, after a 5-sec retention interval, they were shown a test array that was identical or differed in the color of one object. They made an unspeeded forced-choice response at the end of the trial to indicate whether one of the items had changed or not during the retention interval. This task was designed to fill visual working memory to capacity (see Vogel, Woodman, \& Luck, 2001, for a detailed characterization of performance in this task). In the visual search task, the subjects viewed arrays of 4,8 , or 12 squares, each with a gap on one side, and made a speeded forcedchoice response to indicate whether one of the squares had a gap on the top or on the bottom. In the third condition, the subjects performed the search task during the retention interval of the memory task.

The authors reasoned that, if access to visual working memory is crucial for efficient visual search perfor- 
mance, filling visual working memory with information before search began should lead to impaired search efficiency, impaired memory accuracy, or both. However, the efficiency of the subjects' visual search-quantified as the slope of the function relating reaction time (RT) to the number of items in the search array-did not differ between search performed in isolation and search performed during the retention interval of the memory task. The memory load did lead to a significant increase in the $y$-intercept of the search function, but changes in the intercept alone must reflect changes in processes that precede or follow the search process, rather than a change in the efficiency of the search process itself. In addition, accuracy in the memory task was slightly impaired when the search and the memory tasks were performed together, as compared with when the memory task was performed alone, but this effect also did not vary with the number of items in the search array. In other words, visual working memory performance was equally accurate regardless of the number of items that needed to be processed in the search array. Follow-up experiments indicated that this small memory impairment reflected a nonspecific masking of the memory representation by the mere presence of the search array and occurred even if the subjects did not perform a task with the search array. The same pattern of results was obtained when the working memory task required the subjects to remember the shapes of objects that were identical to the objects in the search displays. Overall, strikingly little interference was observed between visual search and visual working memory in this study.

Although the findings of Woodman et al. (2001) demonstrate that maintaining object representations in visual working memory does not impair visual search efficiency, it is possible that maintaining a different type of information could interfere with search. Specifically, a growing body of evidence from behavioral (for reviews, see Baddeley \& Logie, 1999; Logie, 1995), neuroanatomical (for reviews, see Goldman-Rakic, 1996; Smith \& Jonides, 1997), and neuropsychological (e.g., Carlesimo, Perri, Turriziani, Tomaiuolo, \& Caltagirone, 2001; De Renzi \& Nichelli, 1975; Farah, Hammond, Levine, \& Calvanio, 1988) studies supports the proposal that spatial and non- spatial visual representations are stored in separate working memory subsystems. This hypothesis regarding separate working memory subsystems is essentially an extension of the general view that dorsal and ventral pathways in the posterior visual cortex specialize in the processing of where and what, respectively. Thus, in the present study, we examined the possibility that maintaining spatial representations in working memory does interfere with visual search, even though maintaining object representations in visual working memory does not.

To test whether holding spatial locations in working memory interferes with visual search, we used a procedure virtually identical to that used by Woodman et al. (2001). As is shown in Figure 1, the working memory task was changed so that the subjects were required to remember the spatial locations of two objects so that they could perform a location change detection task. We again tested the working memory task alone, the search task alone, and the search task interposed within the retention interval of the working memory task.

If visual search and spatial working memory use a common set of processing resources, search efficiency should be impaired when the search task is presented during the retention interval of the working memory task, as compared with when the search task is tested in isolation. The impairment in search efficiency might also be accompanied by a decrement in memory performance that increases as the number of items to be searched increases. However, if spatial working memory and visual search do not use common processing resources, no such effects should be seen, as Woodman et al. (2001) observed for object memory.

\section{METHOD}

\section{Subjects}

Twelve University of Iowa students with normal or corrected-tonormal vision participated after informed consent was obtained. They reported no history of neurological problems and were paid $\$ 8$ per hour. All the subjects reported being right-handed.

\section{Stimuli and Procedure}

The stimuli were presented on a video monitor with a gray background $\left(0.62 \mathrm{~cd} / \mathrm{m}^{2}\right)$ and a continuously visible fixation cross at a

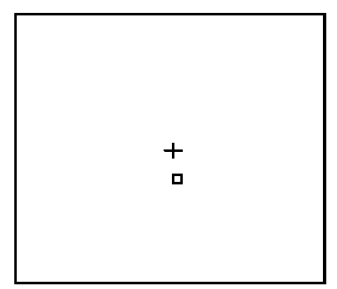

$500 \mathrm{msec}$

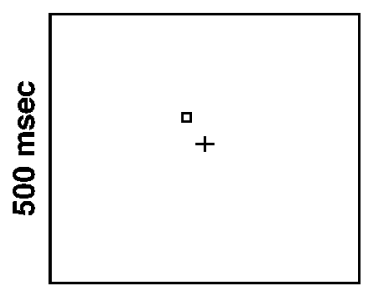

$500 \mathrm{msec}$

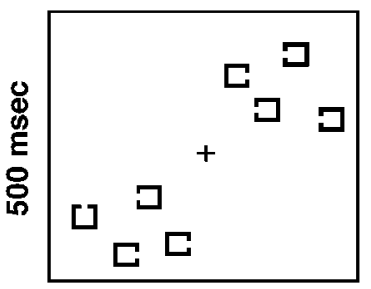

$4,000 \mathrm{msec}$

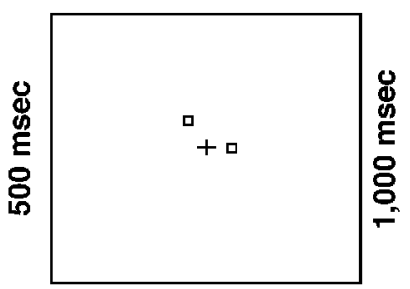

$2,000 \mathrm{msec}$

Figure 1. Example of a single trial in the dual-task condition: The correct response for the memory task would be different, because the location of the dot below fixation changed. Note that this example is shown in reversed contrast; the actual stimuli were white on a dark gray background. 
viewing distance of $70 \mathrm{~cm}$. Each subject completed four blocks of trials, one for the memory task alone, one for the search task alone, and two for the dual-task condition. Each block consisted of 72 trials, preceded by 1015 practice trials. The order of the three conditions was counterbalanced across subjects.

In the memory-only condition, the subjects performed a location change detection task. Each trial began with the sequential presentation of two white dots $\left(0.13^{\circ} \times 0.13^{\circ}, 65.38 \mathrm{~cd} / \mathrm{m}^{2}\right)$ that were used to indicate the to-be-remembered locations. Each dot was presented for $500 \mathrm{msec}$, and the two dots were separated by a 500 -msec blank period. The dots were presented sequentially to discourage observers from forming a shape-based conf igural representation of the two locations (e.g., a line of a certain orientation). Each dot was presented at a randomly selected location within a $1.625^{\circ} \times 1.625^{\circ}$ region centered in the middle of the display, and each dot was centered at least $0.49^{\circ}$ from the center of the other dot's location and from the fixation cross.

The offset of the second dot was followed by a 5,000-msec retention interval and then by a test array in which both dots were shown simultaneously for $2,000 \mathrm{msec}$. On $50 \%$ of the trials, the two dots were shown at the same locations as during the initial presentation. On the other $50 \%$ of the trials, one of the dots was presented at a new location that was centered at least $0.49^{\circ}$ from the original dot, from the other dot, and from the fixation cross. The observers made an unspeeded two-alternative response to indicate whether a location change was detected. The subjects responded on a game pad, pressing a button with the index finger of the left hand to indicate same and pressing a button with the middle finger of the left hand to indicate different. Each trial was followed by a 1,000-msec intertrial interval (ITI).

In the search-only condition, the subjects performed a search task for two possible target shapes, one of which was present on each trial (this is exactly the same task as that used by Woodman et al., 2001). Each search array was composed of 4,8 , or 12 white outlined squares $\left(0.45^{\circ} \times 0.45^{\circ}, 0.08^{\circ}\right.$ line thickness, $\left.65.38 \mathrm{~cd} / \mathrm{m}^{2}\right)$, each of which contained a gap $\left(0.12^{\circ}\right)$ on one side. The items were presented at randomized locations within a $6.1^{\circ} \times 6.1^{\circ}$ region, with each item being at least $1.14^{\circ}$ from the center of the display and at least $0.6^{\circ}$ from the nearest neighboring item (measured from center to center). Note that the region in which search items were presented excludes the central $2.28^{\circ} \times 2.28^{\circ}$ of the display so as not to overlap with the region in which the memory items appeared. Array density was controlled across set sizes by presenting clusters of four items within a single quadrant of the display and varying the number of quadrants containing a cluster. That is, one cluster was presented for a set size of 4, two clusters were presented for a set size of 8 , and three clusters were presented for a set size of 12. Each array was presented for 4,000 msec, separated by a 1,000-msec ITI. Nontarget squares had gaps on the left or the right side, and the target square had a gap on either the top or the bottom of the square. The subjects made a speeded two-alternative response to indicate which of the two possible targets was present on each trial (this twotarget procedure discouraged the subjects from "giving up" before searching the entire array, thus minimizing speed accuracy tradeoffs). They pressed a button with the index finger of the right hand to indicate that a target with a gap on its top was present, and they pressed a button with the middle finger of the right hand to indicate that the target had a gap on its bottom.

In the dual-task condition, the subjects performed both the spatial memory task and the search task on each trial. As is shown in Figure 1, the subjects were shown the visual search array during the retention interval of the memory task. Note that the speeded search responses were made with the right hand and the unspeeded memory responses were made with the left hand. A 500-msec interstimulus interval (ISI) separated the offset of the second dot in the memory task from the onset of the visual search array, and another 500-msec ISI separated the offset of the search array from the onset of the memory test array.
In all the conditions, the subjects performed an articulatory suppression task throughout each trial. In particular, they repeated " $a, b$, c, d," " $1,2,3,4$," "w, x, y, z" or " $6,7,8,9$ " aloud at a rate of 34 items per second during each entire trial. The same set of four letters or digits was repeated on every trial for a block of trials, and the order of the verbal load sets across blocks was randomly selected for each subject. Articulatory suppression tasks such as this have been shown to be highly effective in preventing subjects from recoding visual information for storage in the verbal working memory system (Baddeley, 1986; Besner, Davies, \& Daniels, 1981; Murray, 1968).

\section{RESULTS}

We first will consider the data from the visual search task. As is shown in Figure 2A, RTs increased linearly as set size increased in both the search-alone and the dualtask conditions. However, the slope of the search function was greater in the dual-task condition $(74.4 \mathrm{msec} /$ item) than in the search-alone condition ( $52.9 \mathrm{msec} /$ item $)$. The $y$-intercept was also greater in the dual-task condition $(698 \mathrm{msec})$ than in the search-alone condition $(583 \mathrm{msec})$. These observations were supported by an analysis of variance (ANOVA) with factors of condition (search alone or dual task) and set size $(4,8$, or 12$)$. This analysis yielded a significant main effect of condition $[F(1,11)=50.31, p<.0001]$, a significant main effect of set size $[F(2,22)=151.41, p<.0001]$, and a significant interaction between these factors $[F(2,22)=38.24$, $p<.0001]$.

Accuracy was above $97 \%$ correct at every set size in the search-alone and the dual-task conditions and did not change systematically across the cells of the design. An ANOVA with factors of condition and set size yielded no significant main effects or interactions (all $p \mathrm{~s}>.40$ ).

Accuracy for the spatial memory task is summarized in Figure 2B. Accuracy was quantified as percentage correct, pooling across change and no-change trials (an identical pattern of results was observed for the $A^{\prime}$ measure of detection sensitivity). Mean accuracy was $95 \%$ correct in the memory-alone condition and $82 \%$ correct in the dual-task condition (averaged across the three search set sizes), a significant difference $[t(11)=19.03$, $p<.01]$. In the dual-task condition, memory accuracy decreased as the set size of the concurrent visual search task increased. This observation was supported by a significant one-way ANOVA comparing change detection accuracy across the three search set sizes of the dual-task condition $[F(1,11)=7.00, p<.01]$.

This pattern of results is quite different from the pattern observed by Woodman et al. (2001), who combined this same search task with a color or form change detection task. In the previous study, no set-size dependent interference was observed: The intercept, but not the slope, of the search function was increased in the dual-task condition, and there was no set-size dependent impairment in memory performance in the dual-task condition. In contrast, combining the search task with a location change detection task in the present experiment yielded a significant increase in the slope of the search function and a set-size dependent decline in memory accuracy. 

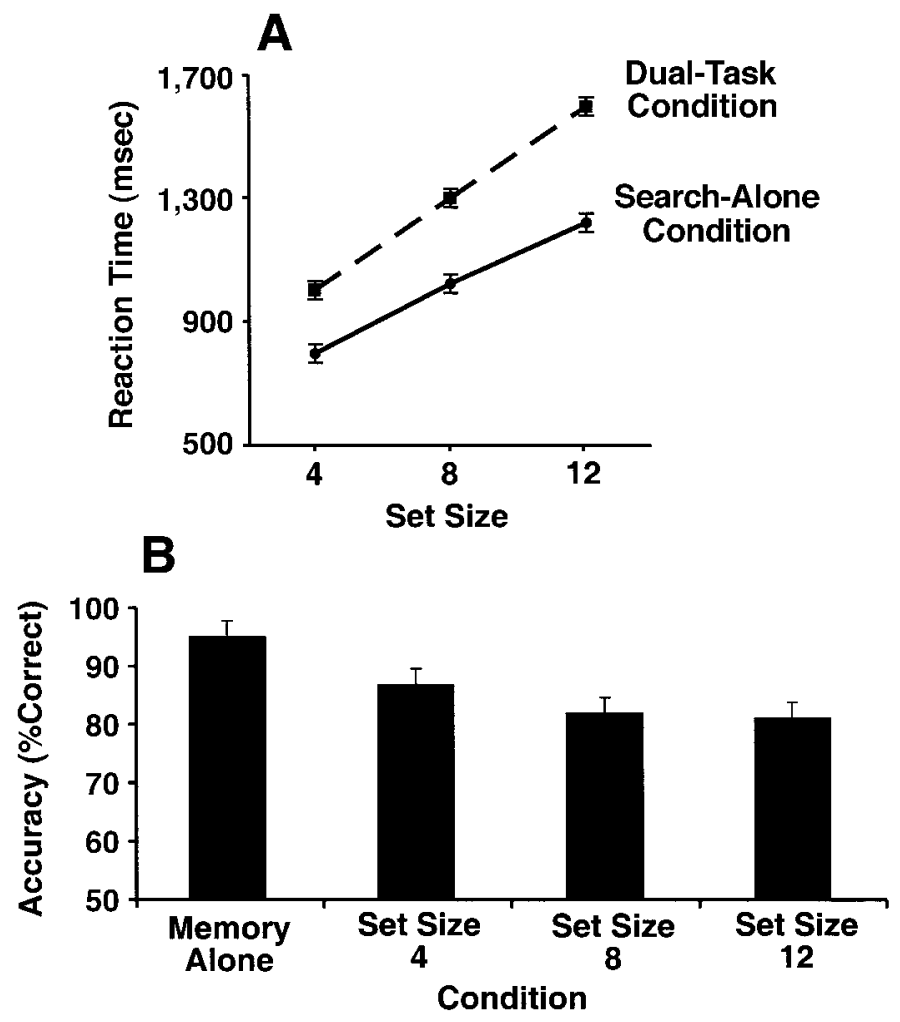

Figure 2. (A) Visual search reaction times with and without spatial working memory load. (B) Visual working memory accuracy (percentage correct) with and without search during the retention interval. Error bars show $95 \%$ within-subjects confidence intervals (Loftus \& Loftus, 1988).

\section{DISCUSSION}

In this study, we investigated the effects of maintaining spatial information in visual working memory on a concurrently performed visual search task. We found that maintaining a relatively modest number of spatial locations interfered with the efficiency of visual search. In addition, memory accuracy became progressively more impaired as the number of items in the search array increased. Thus, the memory task interfered with search performance, and the search task interfered with memory performance; importantly, both types of interference increased as the set size of the search array increased.

No set-size dependent interference effects had been found previously when a color or form change detection task was used instead of a location change detection task, even when the color or form change detection tasks were more difficult than the present location change detection task (Woodman et al., 2001). Together, these two studies indicate that visual search and spatial change detection both require access to a common limited-capacity process, whereas visual search and color and form change detection do not.
What is the common limited-capacity process that is required by the visual search and the spatial change detection tasks? There are several possibilities, the most obvious of which is visual-spatial attention. Specifically, Awh, Jonides, and colleagues (Awh \& Jonides, 1998; Awh, Jonides, \& Reuter-Lorenz, 1998; Jonides \& Smith, 1997; Jonides et al., 1993; Smith \& Jonides, 1997; Smith et al., 1995) have proposed that representations of spatial locations are maintained in working memory by focusing visual-spatial attention on the to-be-remembered locations. We have previously shown that the visual search task used in the present study involves sequential shifts of spatial attention (Woodman \& Luck, 1999), and it is likely that the subjects could not simultaneously attend to the to-be-remembered locations and shift attention from item to item in the search array.

A related possibility is that both the search and the change detection tasks required access to a common system for representing spatial locations. Representations of location are obviously necessary for the change detection task, but they may also be necessary for controlling the operation of attention in the search task. Thus, even if attention is not used for maintaining the spatial locations in 
working memory, the common use of spatial representations may have led to interference between the tasks. By analogy, bilateral parietal lesions lead to both spatial impairments and impairments in attention-demanding visual search tasks (e.g., Robertson, Treisman, FriedmanHill, \& Grabowecky, 1997), and it is difficult to determine whether the attention problems are a result of a more general problem with spatial representations.

Yet another possibility is that visual working memory is used to maintain a record of which locations have been searched so that objects are not searched multiple times (Klein, 1988). If working memory is unavailable for this purpose, search may become less efficient (and if working memory is being used for this purpose, performance on the change detection task would become impaired). The evidence for this sort of memory-guided search is mixed (e.g., Mueller \& von Muehlenen, 2000; Wolfe \& Pokorny, 1990), but even if subjects do not remember exactly which items have been searched, spatial working memory may be used to promote a general systematicity in search (e.g., a general top bottom, left right, or in out search order), and this systematicity may have been compromised by the concurrent spatial change detection task.

The present results also have important implications for the nature of visual working memory. Specifically, the observation that maintaining spatial locations, but not object representations, in working memory impairs visual search provides further support for existing proposals that location and object information are handled by separate working memory subsystems (e.g., Baddeley \& Logie, 1999). Our results parallel previous results reported by Logie and Marchetti (1991), who combined either a temporal spatial memory task or a brightness memory task with either a spatial movement task or the viewing of line drawings. The spatial movement task interfered with performance of the temporal spatial memory task, but not of the brightness memory task, whereas the line drawings interfered with performance of the brightness memory task but not of the temporal spatial memory task. Thus, the present results converge well with previous evidence for functionally separable spatial and object working memory subsystems.

\section{REFERENCES}

AWH, E., \& JONIDES, J. (1998). Spatial working memory and spatial selective attention. In R. Parasuraman (Ed.), The attentive brain (pp. 353-380). Cambridge, MA: MIT Press.

Awh, E., Jonides, J., \& Reuter-Lorenz, P. A. (1998). Rehearsal in spatial working memory. Journal of Experimental Psychology: Human Perception \& Performance, 24, 780-790.

BADDELEY, A. D. (1986). Working memory. Oxford: Oxford University Press, Clarendon Press.

BAdDE LEy, A. D., \& Logie, R. H. (1999). Working memory: The multiple component model. In P. Shah \& A. Miyake (Eds.), Models of working memory (pp. 28-61). Cambridge: Cambridge University Press.

BesneR, D., DAVIES, J., \& DANIELS, S. (1981). Reading for meaning: The effects of concurrent articulation. Quarterly Journal of Experimental Psychology, 33A, 415-437.

Bundesen, C. (1990). A theory of visual attention. Psychological Review, 97, 523-547.

Carlesimo, G. A., Perri, R., Turriziani, P., Tomaiuolo, F., \& Cal-
TAGIRONE, C. (2001). Remembering what but not where: Independence of spatial and visual working memory in the human brain. Cortex, 36, 519-534.

CAVE, K. R., \& Bichot, N. P. (1999). Visuospatial attention: Beyond a spotlight model. Psychonomic Bulletin \& Review, 6, 204-223.

DE RENZI, E., \& Nichelli, P. (1975). Verbal and nonverbal short term memory impairment following hemispheric damage. Cortex, 11, 341-354.

Desimone, R., \& Duncan, J. (1995). Neural mechanisms of selective visual attention. Annual Review of Neuroscience, 18, 193-222.

DunCAN, J., \& HumphreYs, G. W. (1989). Visual search and stimulus similarity. Psychological Review, 96, 433-458.

FARAH, M. J., Hammond, K. M., Levine, D. N., \& Calvanio, R. (1988). Visual and spatial mental imagery: Dissociable systems of representation. Cognitive Psychology, 20, 439-462.

GoldMAN-RAKIC, P. S. (1996). Regional and cellular fractionation of working memory. Proceedings of the National Academy of Sciences, 93, 13473-13480.

JoNidEs, J., \& SMITH, E. E. (1997). The architecture of working memory. In M. D. Rugg (Ed.), Cognitive neuroscience (pp. 243-276). Cambridge, MA: MIT Press.

Jonides, J., Smith, E. E., Koepre, R. A., Awh, E., Minoshima, S., \& Mintun, M. A. (1993). Spatial working memory in humans as revealed by PET. Nature, 363, 623-625.

Kahneman, D., Treisman, A., \& GibBS, B. J. (1992). The reviewing of object files: Object-specific integration of information. Cognitive Psychology, 24, 175-219.

KLEIN, R. (1988). Inhibitory tagging system facilitates visual search. Nature, 334, 430-431.

Loftus, G. R., \& Loftus, E. F. (1988). Essence of statistics (2nd ed.). New York: Random House.

LOGIE, R. H. (1995). Visuo-spatialworking memory. Hove, U.K.: Erlbaum.

Logie, R. H., \& MARCHETTI, C. (1991). Visuo-spatial working memory: Visual, spatial or central executive? In R. H. Logie \& M. Denis (Eds.), Mental images in human cognition (pp. 105-115). Amsterdam: North-Holland.

Luck, S. J., Girelli, M., MCDermott, M. T., \& Ford, M. A. (1997). Bridging the gap between monkey neurophysiology and human perception: An ambiguity resolution theory of visual selective attention. Cognitive Psychology, 33, 64-87.

Mueller, H. J., \& VON Muehlenen, A. (2000). Probing distractor inhibition in visual search: Inhibition of return (IOR). Journal of Experimental Psychology: Human Perception \& Performance, 26, 1332-1351.

MurRAY, D. J. (1968). Articulation and acoustic confusability in shortterm memory. Journal of Experimental Psychology, 78, 679-684.

Robertson,L., Treisman, A., Friedman-Hill, S., \& Grabowecky, M. (1997). The interaction of spatial and object pathways: Evidence from Balint's syndrome. Journal of Cognitive Neuroscience, 9, 295-317.

SMith, E. E., \& Jonides, J. (1997). Working memory: A view from neuroimaging. Cognitive Psychology, 33, 5-42.

Smith, E. E., Jonides, J., Koe PPe, R. A., Awh, E., Schumacher, E. H., \& Minoshima, S. (1995). Spatial versus object working memory: PET investigations. Journal of Cognitive Neuroscience, 7, 337-356.

Treisman, A. [M.] (1988). Features and objects: The Fourteenth Bartlett Memorial Lecture. Quarterly Journal of Experimental Psychology, 40A, 201-237.

Treisman, A. M., \& Gelade, G. (1980). A feature-integration theory of attention. Cognitive Psychology, 12, 97-136.

Treisman, A. [M.], \& SATO, S. (1990). Conjunction search revisited. Journal of Experimental Psychology: Human Perception \& Performance, 16, 459-478.

Vogel, E. K., Woodman, G. F., \& Luck, S. J. (2001). Storage of features, conjunctions, and objects in visual working memory. Journal of Experimental Psychology: Human Perception \& Performance, 27, 92-114.

Wolfe, J. M. (1994). Guided Search 2.0: A revised model of visual search. Psychonomic Bulletin \& Review, 1, 202-238.

Wolfe, J. M. (1998). Visual search. In H. Pashler (Ed.), Attention (pp. 13-73). Hove, U.K.: Psychology Press/Erlbaum.

Wolfe, J. M., CAVE, K. R., \& Franzel, S. L. (1989). Guided search: 
An alternative to the feature integration model for visual search. Journal of Experimental Psychology: Human Perception \& Performance, 15, 419-433.

Wolfe, J. M., \& POKORNY, C. W. (1990). Inhibitory tagging in visual search: A failure to replicate. Perception \& Psychophysics, 48, 357-362.

Woodman, G. F., \& LUCK, S. J. (1999). Electrophysiological measurement of rapid shifts of attention during visual search. Nature, 400, 867-869.

Woodman, G. F., Vogel, E. K., \& Luck, S. J. (2001). Visual search remains efficient when visual working memory is full. Psychological Science, 12, 219-224.

\section{NOTE}

1. The term working memory has been used in different ways by different investigators. The task used here presumably relies on the visual storage buffers postulated in Baddeley's (1986) model of working memory, and it is a working memory task in that sense.

(Manuscript received November 5, 2002; revision accepted for publication April 7, 2003.) 